\title{
Bcl-xL Dynamics under the lens of Protein Structure Networks
}

\author{
Valentina Sora ${ }^{1,2}$, Dionisio Sanchez ${ }^{1}$, Elena Papaleo ${ }^{1,2}$
}

${ }^{1}$ Computational Biology Laboratory, Danish Cancer Society Research Center, 2100, Copenhagen, Denmark

${ }^{2}$ Cancer Systems Biology, Section for Bioinformatics, Health and Technology

Department, Technical University of Denmark, 2800, Lyngby, Denmark

Supplementary Figure $S 1$ - Average backbone and $C \beta$ reduced $X^{2}$ values of the $B c l-x_{L}-P U M A$ replicates

Averages of reduced $X^{2}$ values from all backbone and $C \beta$ atoms of all seven Bcl- $x_{L}$-PUMA replicates. In panel $A$, the average metric includes values from all atoms for which data is available. In panel $B$, the average metric is presented excluding the five highest values (out of more than 140 entries) for $C a$ and $C \beta$ atoms consistently found across replicates listed in Supplementary Figure S4. Although we observed high values of the average reduced $X^{2}$ for $C a$ and $C \beta$ atoms, we noticed that these averages were consistently dominated by a reduced group of atoms in all replicates, for which the computed reduced $\mathrm{X}^{2}$ was similar. Interestingly, similar high values of the reduced $X^{2}$ were not found in the remaining backbone atoms of these residues.

A)

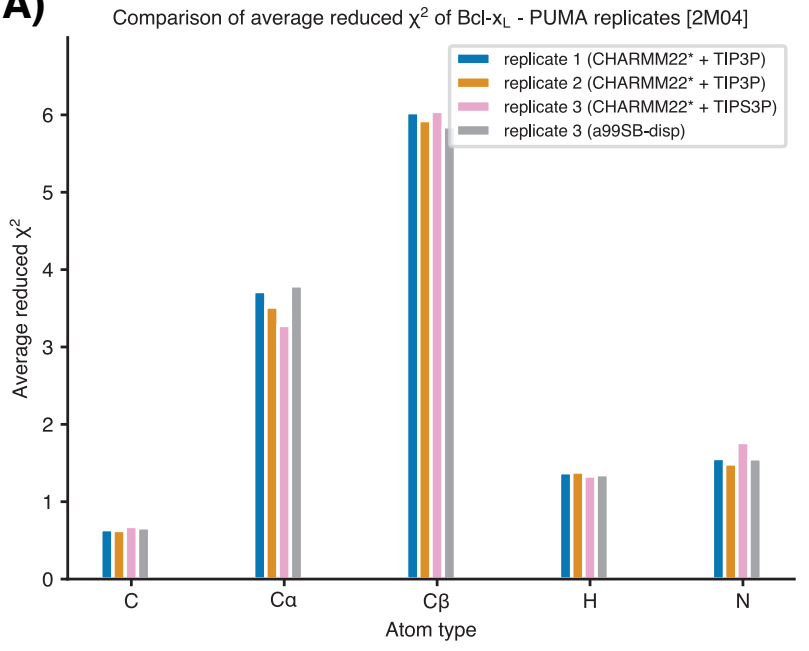

B)

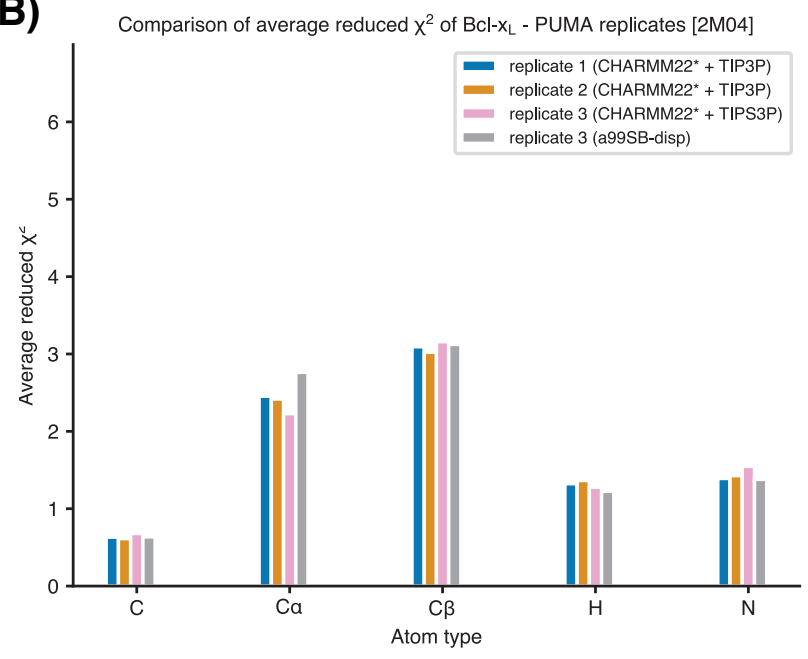




\section{Bcl-xL Dynamics under the lens of Protein Structure Networks}

\section{Valentina Sora ${ }^{1,2}$, Dionisio Sanchez ${ }^{1}$, Elena Papaleo ${ }^{1,2}$}

${ }^{1}$ Computational Biology Laboratory, Danish Cancer Society Research Center, 2100, Copenhagen, Denmark

${ }^{2}$ Cancer Systems Biology, Section for Bioinformatics, Health and Technology

Department, Technical University of Denmark, 2800, Lyngby, Denmark

Supplementary Table $\mathbf{S} 2$ - Table with the five $C a$ and $C \beta$ atoms with highest reduced $X^{2}$ values in the Bcl-xL-PUMA replicates

Table with the five $\mathrm{Ca}$ and $\mathrm{C} \beta$ atoms with highest reduced $\mathrm{X}^{2}$ values in the $\mathrm{Bcl}$-xL-PUMA replicates. The atoms with particularly high reduced $X^{2}$ values distorting the averages from Supplementary Figure S1A are consistent and present similar reduced $\mathrm{X}^{2}$ values across all four replicates.

$\mathrm{Ca}$ and $\mathrm{C} \beta$ atoms with the highest reduced $\mathrm{X}^{2}$ in $\mathrm{Bcl}-\mathrm{X}_{\mathrm{L}}$ - PUMA replicates

\begin{tabular}{|c|c|c|c|c|c|c|}
\hline $\begin{array}{l}\text { Residue } \\
\text { number }\end{array}$ & Residue & Atom & $\begin{array}{c}\text { Replicate1 } \\
\text { (CHARMM22* } \\
\text { + TIP3P) }\end{array}$ & $\begin{array}{c}\text { Replicate2 } \\
\text { (CHARMM22* } \\
\text { + TIP3P) }\end{array}$ & $\begin{array}{c}\text { Replicate3 } \\
\text { (CHARMM22* }^{*} \\
\text { + TIPS3P) }\end{array}$ & $\begin{array}{c}\text { Replicate3 } \\
\text { (a99SB- } \\
\text { disp) }\end{array}$ \\
\hline 143 & PHE & $C \beta$ & 145.68 & 153.36 & 147.11 & 150.53 \\
\hline 113 & HIS & $C \beta$ & 111.37 & 122.10 & 86.92 & 98.39 \\
\hline 105 & PHE & $C \beta$ & 100.02 & 89.25 & 127.35 & 85.56 \\
\hline 115 & THR & $C \beta$ & 49.01 & 48.65 & 52.79 & 41.59 \\
\hline 158 & GLU & $C \beta$ & 33.40 & 24.28 & 6.95 & 25.47 \\
\hline 95 & ASP & $\mathrm{Ca}$ & 51.82 & 47.64 & 58.69 & 47.12 \\
\hline 144 & PHE & $\mathrm{Ca}$ & 47.79 & 53.71 & 27.85 & 15.95 \\
\hline 99 & LEU & $\mathrm{Ca}$ & 41.22 & 40.62 & 68.10 & 51.60 \\
\hline 169 & TRP & $\mathrm{Ca}$ & 24.13 & 0.82 & 2.82 & 13.43 \\
\hline 159 & MET & $\mathrm{Ca}$ & 23.84 & 20.43 & 6.86 & 30.40 \\
\hline
\end{tabular}




\title{
Bcl-xL Dynamics under the lens of Protein Structure Networks
}

\author{
Valentina Sora ${ }^{1,2}$, Dionisio Sanchez ${ }^{1}$, Elena Papaleo ${ }^{1,2}$
}

${ }^{1}$ Computational Biology Laboratory, Danish Cancer Society Research Center, 2100, Copenhagen, Denmark

${ }^{2}$ Cancer Systems Biology, Section for Bioinformatics, Health and Technology

Department, Technical University of Denmark, 2800, Lyngby, Denmark

Supplementary Figure $S 3$ - Local reduced $X^{2}$ values for backbone and $C \beta$ atoms in the $B c l-x_{L}$ (free) replicates with 2LPC

Local reduced $X^{2}$ values for backbone and $C \beta$ atoms in the $B c l-x_{L}$ (free) replicates. The values for the metric were obtained upon comparison with the chemical shift data set 2LPC (BMRB 18250). White spots are locations where no data or no prediction was available. Values over 3 were saturated to 3 for better visualization.
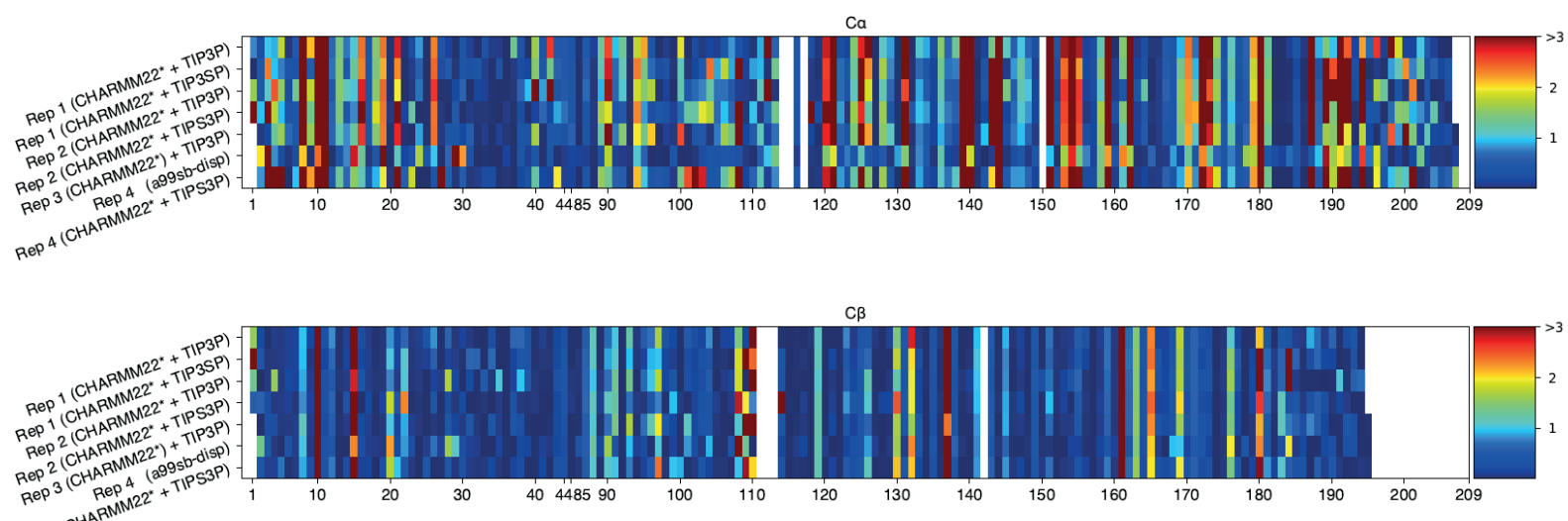
RePA COHAFMM 22
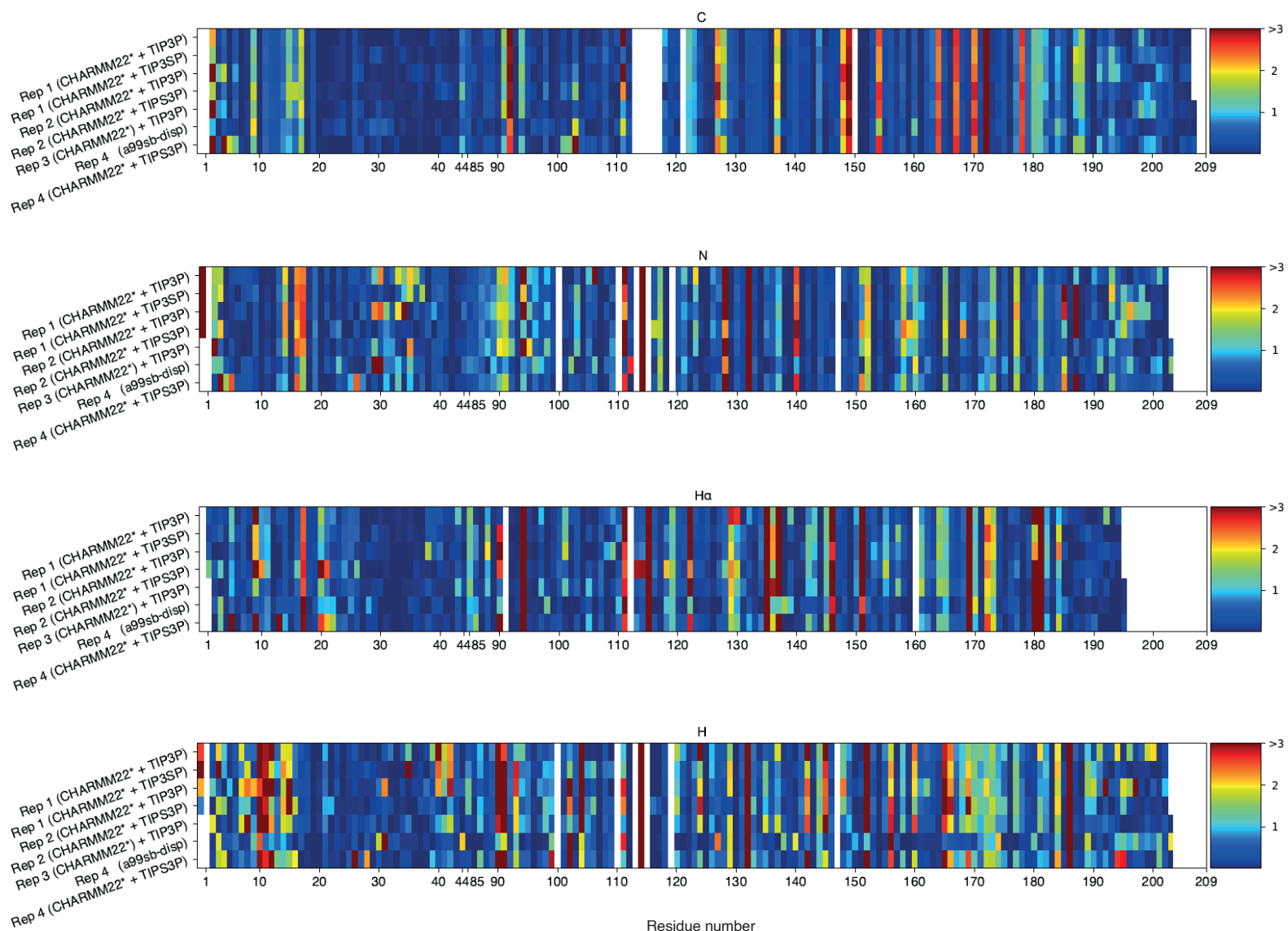


\title{
Bcl-xL Dynamics under the lens of Protein Structure Networks
}

\author{
Valentina Sora ${ }^{1,2}$, Dionisio Sanchez ${ }^{1}$, Elena Papaleo ${ }^{1,2}$ \\ ${ }^{1}$ Computational Biology Laboratory, Danish Cancer Society Research Center, 2100, \\ Copenhagen, Denmark \\ ${ }^{2}$ Cancer Systems Biology, Section for Bioinformatics, Health and Technology \\ Department, Technical University of Denmark, 2800, Lyngby, Denmark
}

Supplementary Figure $\mathbf{S} 4$ - Local reduced $X^{2}$ values for backbone and $C \beta$ atoms in the Bcl- $x_{L}$ (free) replicates with $2 \mathrm{M} 03$

Local reduced $\mathrm{X}^{2}$ values for backbone and $C \beta$ atoms in the $\mathrm{Bcl}-\mathrm{x}_{\mathrm{L}}$ (free) replicates. The values for the metric were obtained upon comparison with the chemical shift data set $2 \mathrm{M03}$ (BMRB 18792). White spots are locations where no data or no prediction was available. Values over 3 were saturated to 3 for better visualization.
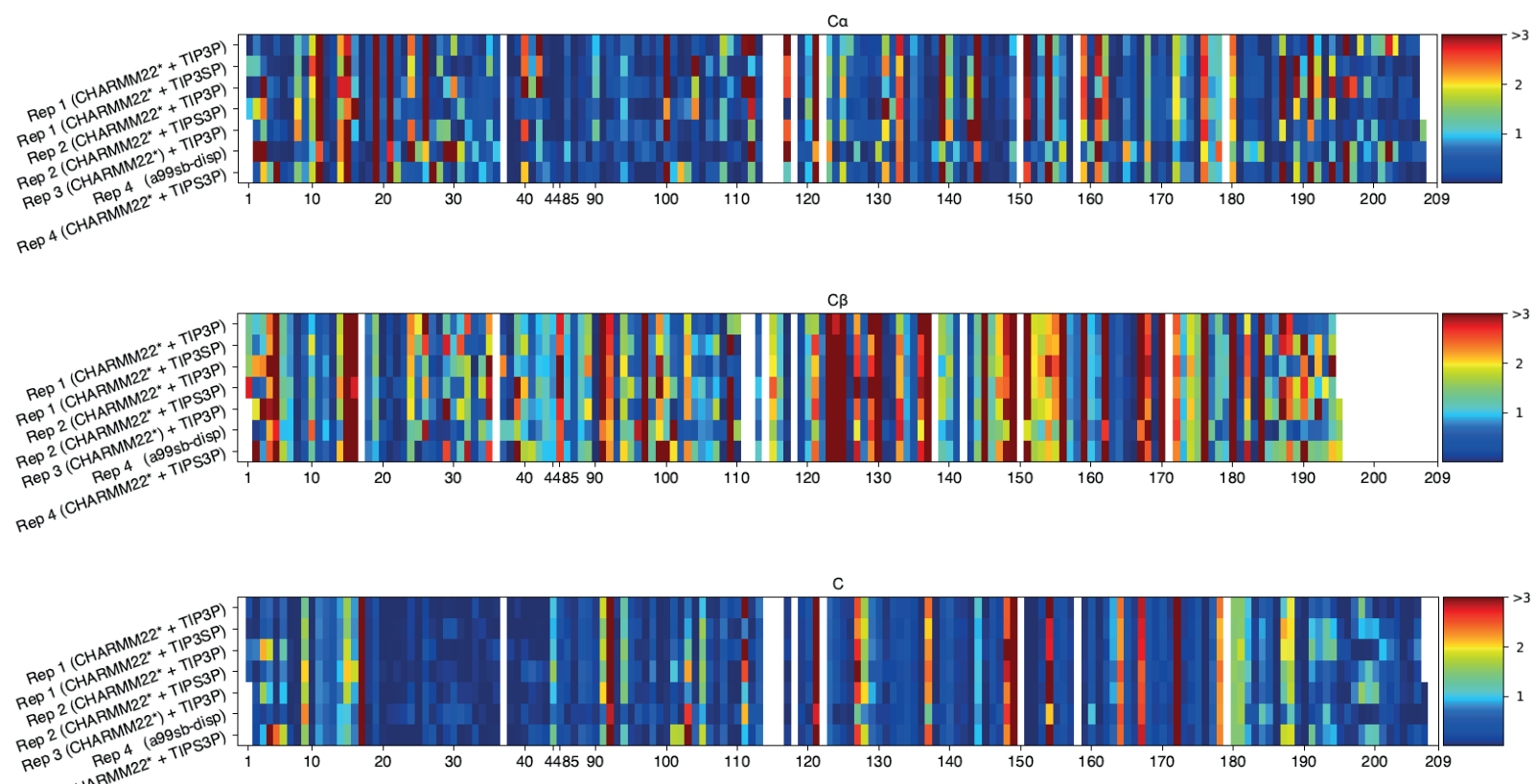
ReP A CHARMM22
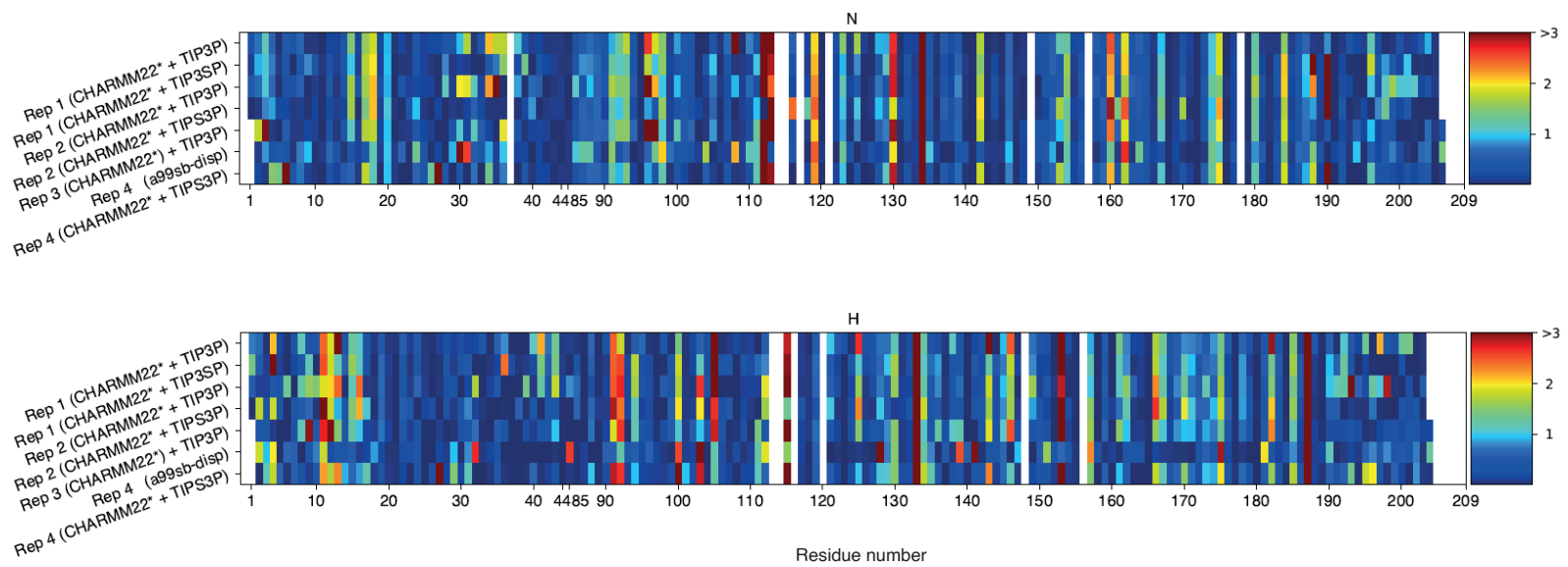


\title{
Bcl-xL Dynamics under the lens of Protein Structure Networks
}

\author{
Valentina Sora ${ }^{1,2}$, Dionisio Sanchez ${ }^{1}$, Elena Papaleo ${ }^{1,2}$ \\ ${ }^{1}$ Computational Biology Laboratory, Danish Cancer Society Research Center, 2100, \\ Copenhagen, Denmark \\ ${ }^{2}$ Cancer Systems Biology, Section for Bioinformatics, Health and Technology \\ Department, Technical University of Denmark, 2800, Lyngby, Denmark
}

Supplementary Figure S5 - Local reduced $X^{2}$ values for backbone and C $\beta$ atoms in the Bcl- $\mathrm{x}_{\mathrm{L}}-\mathrm{PUMA}$ replicates with $2 \mathrm{M} 04$

Local reduced $\mathrm{X}^{2}$ values for backbone and $\mathrm{C} \beta$ atoms in the $\mathrm{Bcl}-\mathrm{x}_{\mathrm{L}}$ - PUMA replicates. The values for the metric were obtained upon comparison with the chemical shift data set 2M04 (BMRB 18793). White spots are locations where no data or no prediction was available. Values over 3 were saturated to 3 for better visualization.
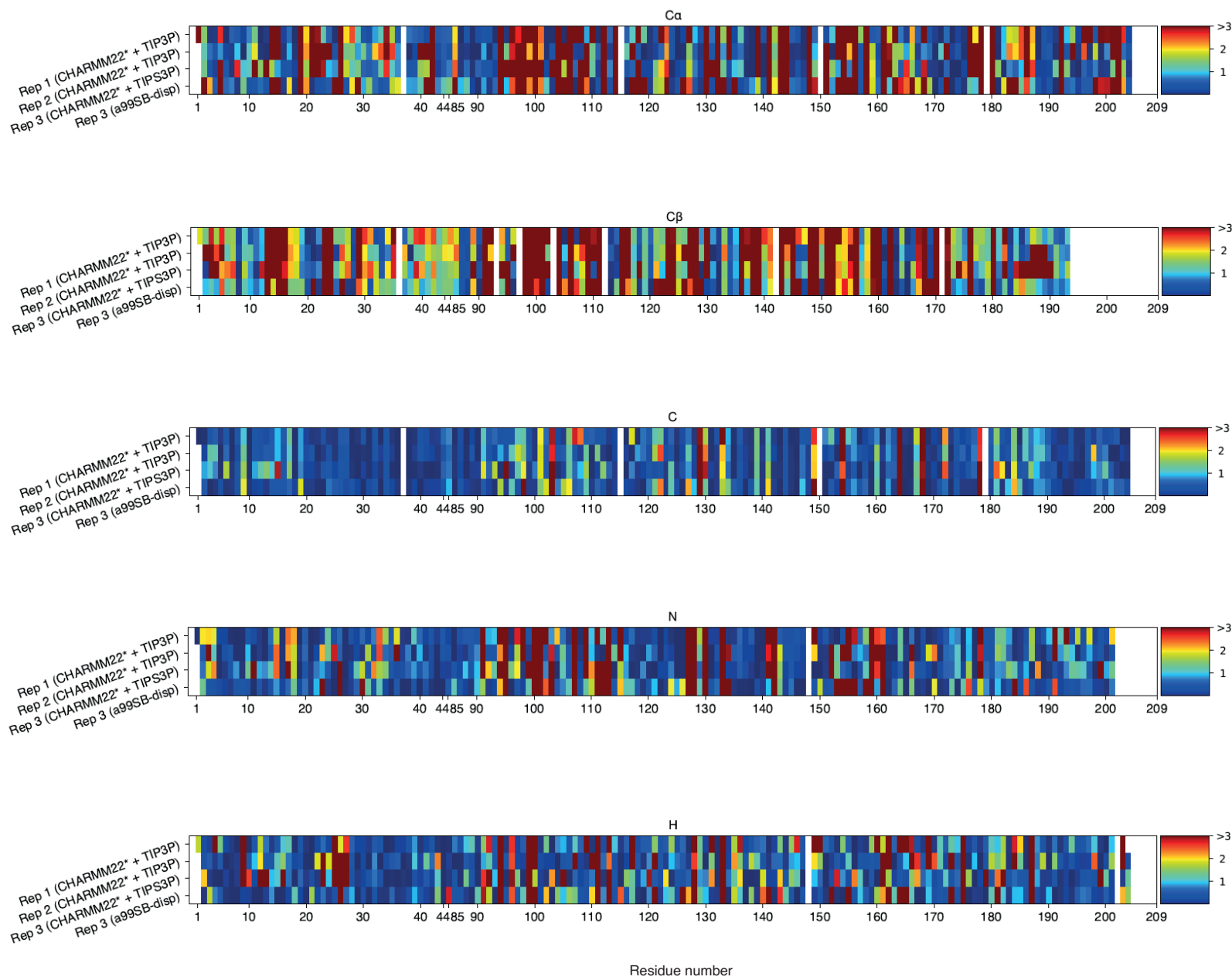


\title{
Bcl-xL Dynamics under the lens of Protein Structure Networks
}

\author{
Valentina Sora ${ }^{1,2}$, Dionisio Sanchez ${ }^{1}$, Elena Papaleo ${ }^{1,2}$ \\ ${ }^{1}$ Computational Biology Laboratory, Danish Cancer Society Research Center, 2100, \\ Copenhagen, Denmark \\ ${ }^{2}$ Cancer Systems Biology, Section for Bioinformatics, Health and Technology \\ Department, Technical University of Denmark, 2800, Lyngby, Denmark
}

Supplementary Figure S6 - Average reduced $X^{2}$ values for methyl $C$ in the Bcl- $x_{L}$ (free) replicates.

Average reduced $\mathrm{X}^{2}$ values for the methyl $\mathrm{C}$ of all seven $\mathrm{Bcl}-\mathrm{x}_{\mathrm{L}}$ (free) replicates. The average values for the metric were obtained upon comparison with the chemical shift data sets 2LPC (BMRB 18250, panel A) and $2 \mathrm{M03}$ (BMRB 18792, panel B). Except for the $\mathrm{CY}_{2}$ of threonine residues in 5 of the 7 replicates, values for the average reduced $\mathrm{X}^{2}$ values around and below two suggest good agreement of chemical shift predictions with chemical shift data.
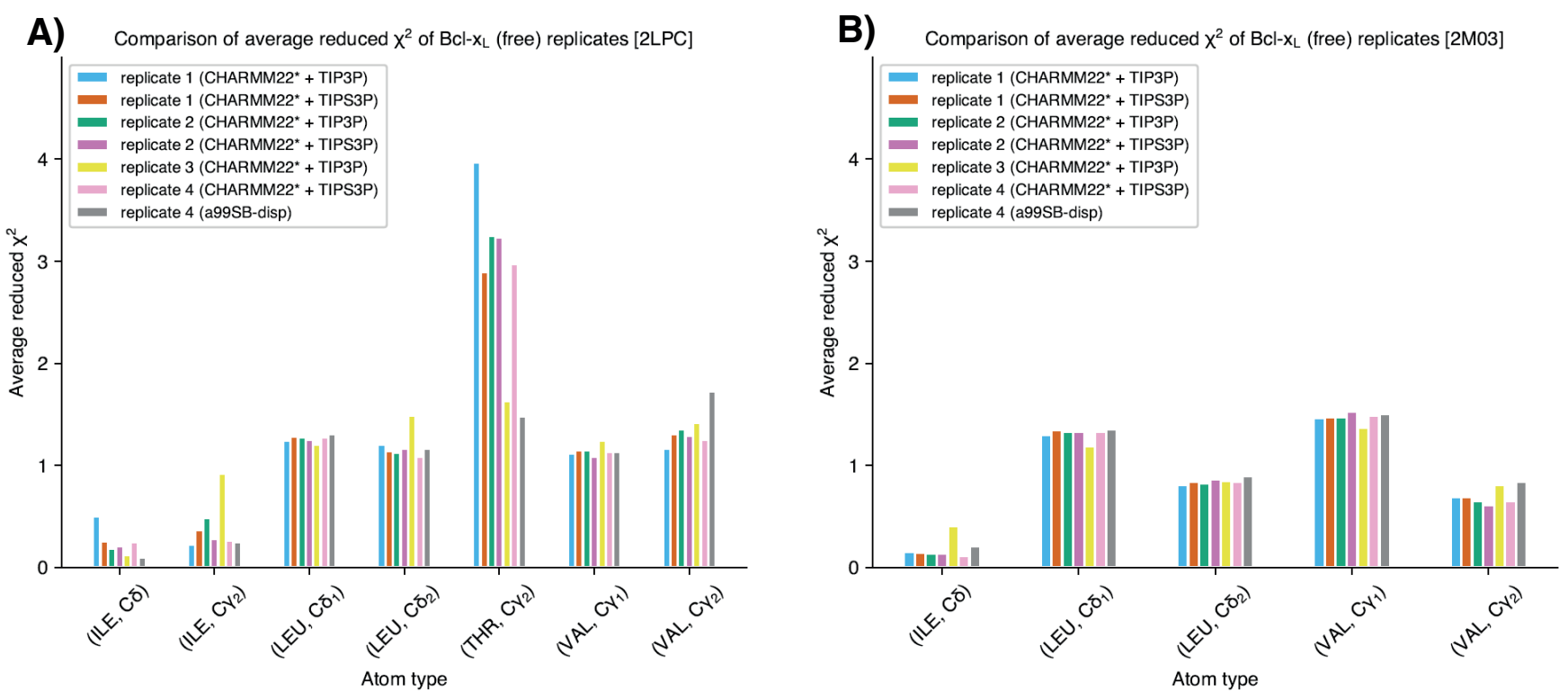


\title{
Bcl-xL Dynamics under the lens of Protein Structure Networks
}

\author{
Valentina Sora ${ }^{1,2}$, Dionisio Sanchez ${ }^{1}$, Elena Papaleo ${ }^{1,2}$ \\ ${ }^{1}$ Computational Biology Laboratory, Danish Cancer Society Research Center, 2100, \\ Copenhagen, Denmark \\ ${ }^{2}$ Cancer Systems Biology, Section for Bioinformatics, Health and Technology \\ Department, Technical University of Denmark, 2800, Lyngby, Denmark
}

Supplementary Figure S7 - Average reduced $\mathrm{X}^{2}$ values for methyl C in the Bcl- $\mathrm{x}_{\mathrm{L}}$ - PUMA replicates.

Average reduced $\mathrm{X}^{2}$ values for the methyl $\mathrm{C}$ of all four $\mathrm{Bcl}-\mathrm{x}_{\mathrm{L}}-\mathrm{PUMA}$ replicates. The average values for the metric were obtained upon comparison with the chemical shift data set 2M04 (BMRB 18793). Except for Leu $\mathrm{C}_{1}$ atoms, where the average is mostly dominated by the residues L8, L112 and L130 in all cases, averages around and below two suggest good agreement of predicted chemical shift with experimental data.

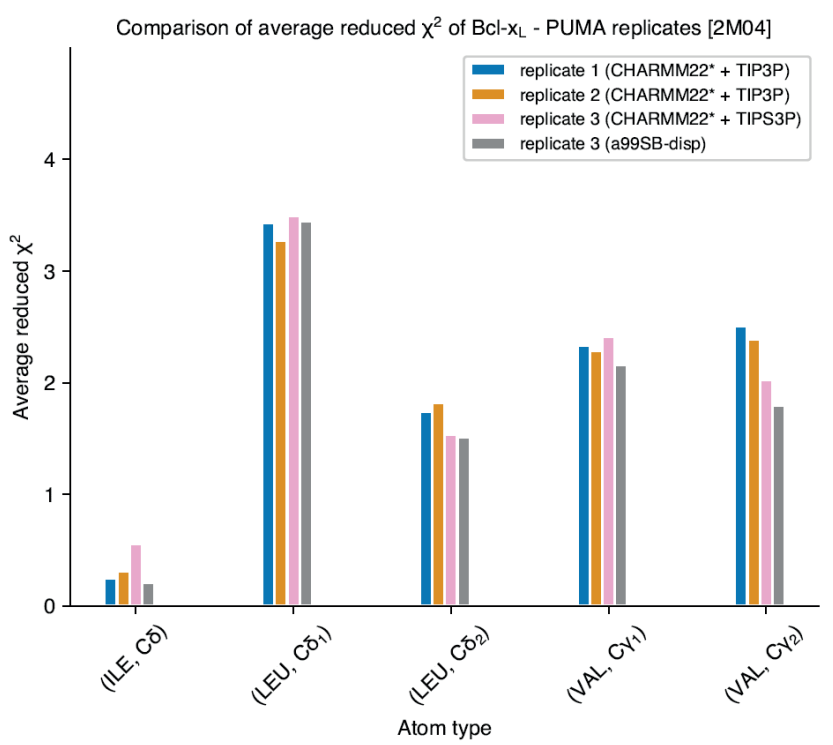




\title{
Bcl-xL Dynamics under the lens of Protein Structure Networks
}

\author{
Valentina Sora',2, Dionisio Sanchez ${ }^{1}$, Elena Papaleo ${ }^{1,2}$ \\ ${ }^{1}$ Computational Biology Laboratory, Danish Cancer Society Research Center, 2100, \\ Copenhagen, Denmark \\ ${ }^{2}$ Cancer Systems Biology, Section for Bioinformatics, Health and Technology \\ Department, Technical University of Denmark, 2800, Lyngby, Denmark
}

\section{Supplementary Figure S8 - Distributions of nodes at different distance cut-offs}

Distribution of nodes in the five most populated connected components in one PSN of Bcl-xL free when the distance cut-off was set to $5 \AA$ (panel A), $5.125 \AA$ (panel B), $5.25 \AA$ (panel C). We chose $5.125 \AA$ as distance cut-off to build our final PSNs since it was a good compromise between an unrealistically sparse network $(5.0 \AA)$ and a hyper-connected one $(5.25 \AA)$.

A)

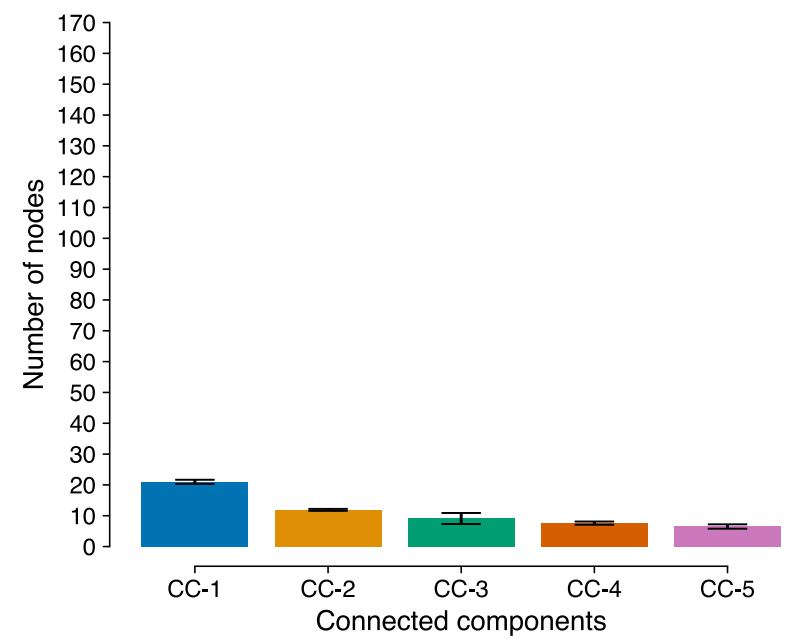

B)

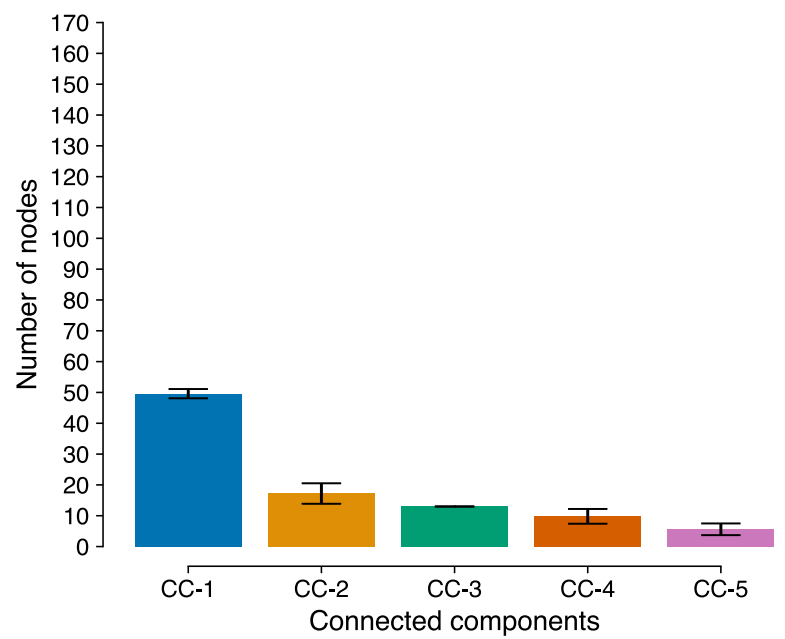

C)

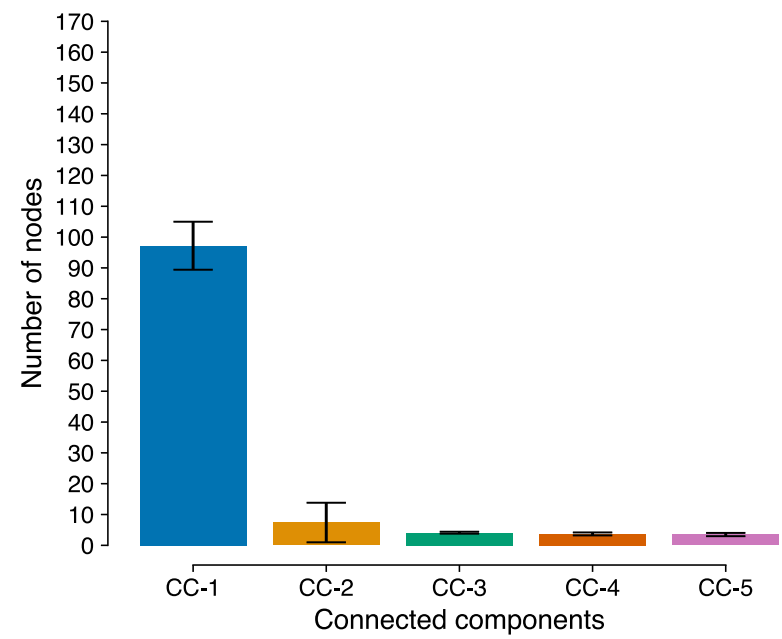




\section{Bcl-xL Dynamics under the lens of Protein Structure Networks}

Valentina Sora ${ }^{1,2}$, Dionisio Sanchez ${ }^{1}$, Elena Papaleo ${ }^{1,2}$

${ }^{1}$ Computational Biology Laboratory, Danish Cancer Society Research Center, 2100, Copenhagen, Denmark

${ }^{2}$ Cancer Systems Biology, Section for Bioinformatics, Health and Technology Department, Technical University of Denmark, 2800, Lyngby, Denmark

\section{Supplementary Figure S9 - All shortest paths}

Schematic representation of all shortest paths found between residues in the p53 binding site (Y22, S23, Q26, S28, V155) and residues in the BH3-only proteins binding site (L108, H113, F146). Lines in green, yellow and pink represent paths found in the PSNs of Bcl- $x_{L}$ free, while blue lines represent paths found in one of the PSNs of Bcl- $x_{L}-P U M A$. In the other PSN of Bcl- $x_{L}-P U M A$, no shortest paths were found between the pairs of residues we were interested in. 
A) Shortest paths from residues in the p53 binding site to L108 (maximum length: 15 residues)
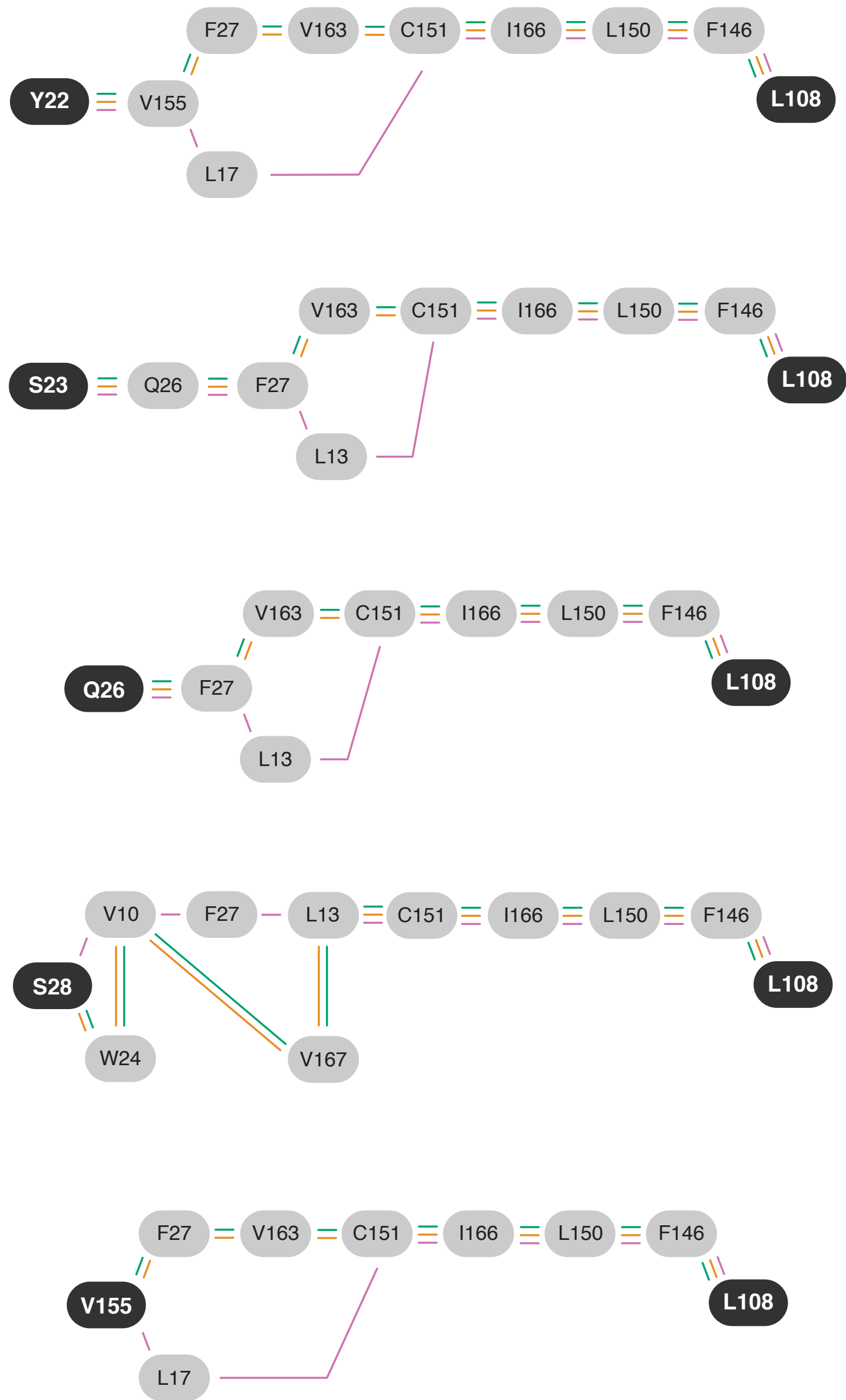
B) Shortest paths from residues in the p53 binding site to H113 (maximum length: 15 residues)

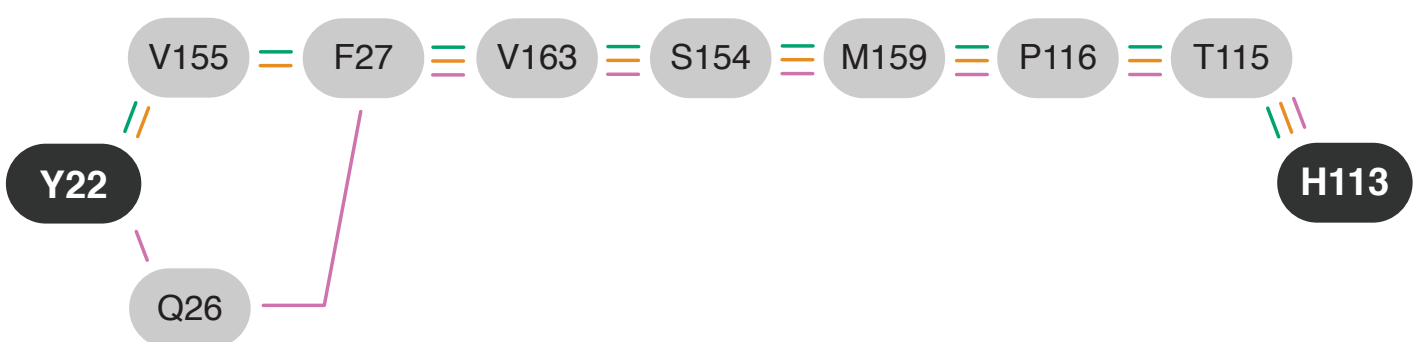

S23 $\equiv \mathrm{Q} 26 \equiv \mathrm{F} 27 \equiv \mathrm{V} 163 \equiv \mathrm{S} 154 \equiv \mathrm{M} 159 \equiv \mathrm{P} 116 \equiv \mathrm{T} 115 \equiv \mathrm{H} 113$

Q26 $\equiv \mathrm{F} 27 \equiv \mathrm{V} 163 \equiv \mathrm{S} 154 \equiv \mathrm{M} 159 \equiv \mathrm{P} 116 \equiv \mathrm{T} 115 \equiv \mathrm{H} 113$

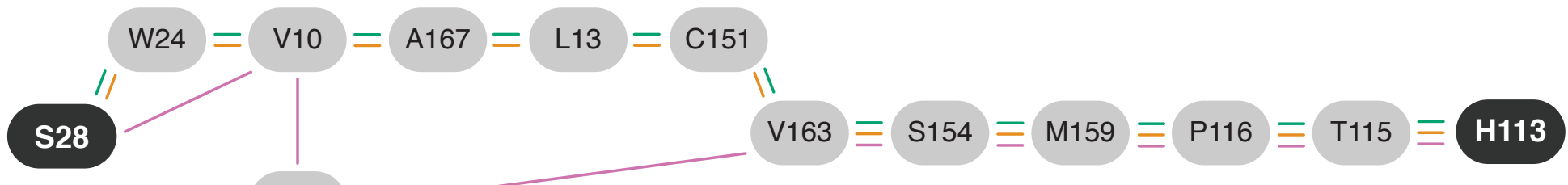

F27

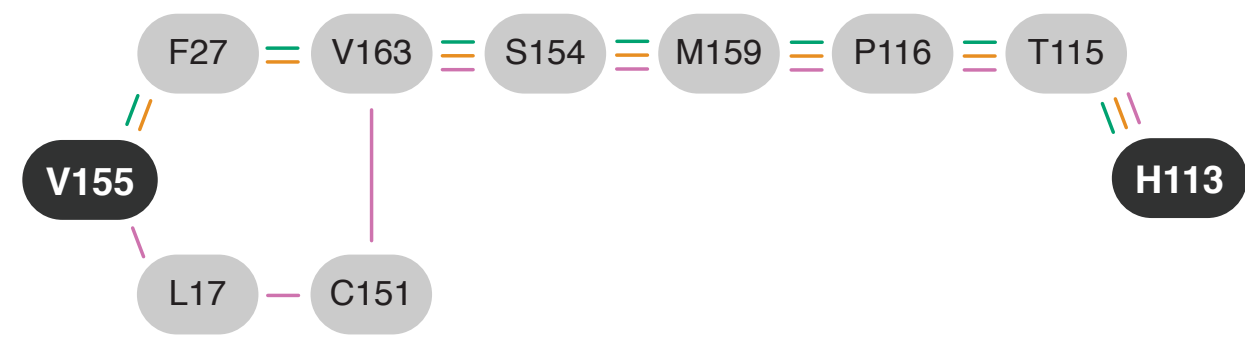



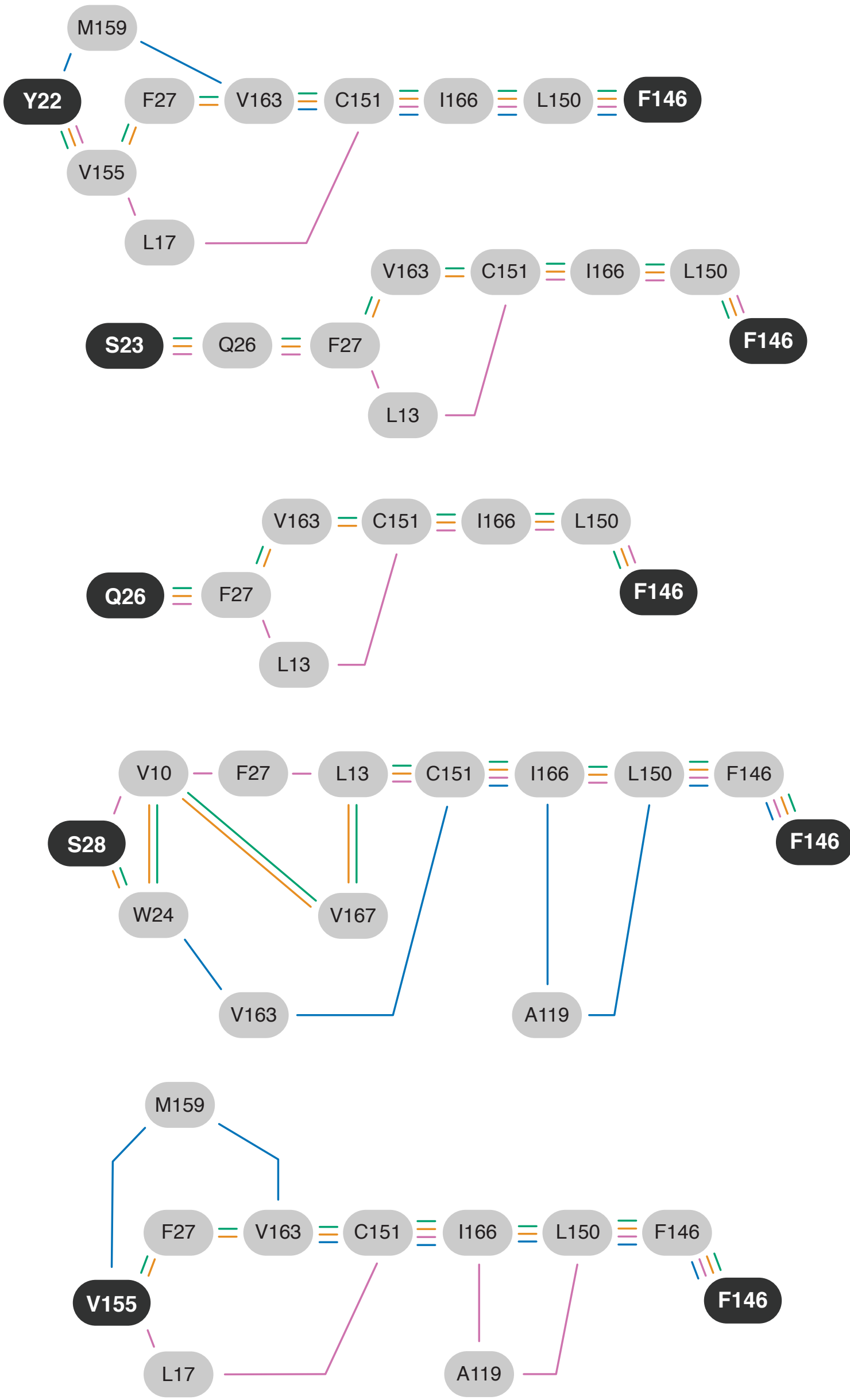